Rev. Bras. Saúde Prod. Anim., Salvador, v.16, n.2, p.290-299 abr./jun.., 2015 http://www.rbspa.ufba.br

\title{
Fatores ambientais e parâmetros genéticos para características reprodutivas de fêmeas Nelore na região meio norte do Brasil
}

\author{
Environmental factors and genetic parameters for reproductive characteristics of \\ Nelore in the through northern region from Brazil
}

\author{
SOUSA, Gioto Ghiarone Terto e ${ }^{1}$; SANTOS, Karina Rodrigues dos ${ }^{2}$; CAMPELO, José \\ Elivalto Guimarães ${ }^{3}$; OLIVEIRA, Marcelo Richelly Alves de ${ }^{3 *}$; PIRES, Luanna \\ Chácara $^{5}$; PEREIRA, Rodrigo Junqueira ${ }^{6}$; SOUSA, Rogério Paes Ribeiro de ${ }^{4}$; SOUSA \\ JÚNIOR, Severino Cavalcante de ${ }^{2}$
}

\footnotetext{
${ }^{1}$ Universidade Estadual do Piauí, Centro de Ciências Agrárias, Teresina, Piauí, Brasil.

${ }^{2}$ Universidade Federal do Piauí, Campus Ministro Reis Velloso, Parnaíba, Piauí, Brasil.

${ }^{3}$ Universidade Federal do Piauí, Centro de Ciências Agrárias, Teresina, Piauí, Brasil.

${ }^{4}$ Universidade Federal do Piauí, Campus Professora Cinobelina Elvas, Departamento de Zootecnia, Bom Jesus, Piauí, Brasil.

${ }^{5}$ Universidade Federal do Sul da Bahia - UFSB, Campus Paulo Freire, Teixeira de Freitas, Bahia, Brasil.

${ }^{6}$ Universidade Federal do Mato Grosso, Grupo de Melhoramento Animal do Mato Grosso, Rondonópolis, Mato Grosso, Brasil.

*Endereço para correspondência: marcelo-zootec@hotmail.com
}

\section{RESUMO}

O estudo foi conduzido para avaliar a idade ao primeiro parto (IPP) e o intervalo de partos (IDP) em fêmeas da raça Nelore utilizando 23.066 registros de IPP e IDP de fêmeas criadas em regime de pasto nos estados do Maranhão e Piauí no período de 1976 a 2012. As análises foram realizadas utilizando modelo animal uni e bicaracterísticas para IPP e IDP. As médias obtidas e os coeficientes de variação para as características de idade ao primeiro parto foram $1252,77 \pm 125$ dias (41,76 meses) a $10,56 \%$; intervalo de partos $591,729 \pm 149,91$ dias $(19,72$ meses) a 38,74\%, respectivamente. Foram estimados parâmetros genéticos em análise unicaracterística, sendo obtidos valores de herdabilidade em torno de 0,026 e 0,006 para IPP e IDP, respectivamente. A estimativa de repetibilidade para as características IDP em análise unicaracterística apresentou-se, de forma geral, com baixa magnitude 0,025 . Na mesma ordem, para análise bicaracterística, as herdabilidades foram de 0,03 e 0,06 para IPP e IDP, e o coeficiente de repetibilidade estimado de 0,14 , respectivamente. As correlações genéticas entre as características reprodutivas (IPP e IDP) observadas neste trabalho foram iguais a 0,06 . O desempenho reprodutivo do rebanho estudado, para as condições de clima tropical, pode ser considerado satisfatório, estando em acordo com os parâmetros encontrados por outros autores em diferentes regiões.

Palavras-chave: bovino de corte, correlação genética, desempenho reprodutivo

\section{SUMMARY}

The study was carried out to evaluate the age-atfirst-calving and calving interval (IPP and IDP) in Nelore cows, using IPP and IDP data from 23,066 females reared in a grazing regime in the States of Maranhão and Piauí from 1976 to 2012. Data were analyzed statistically using the SAS (Statistical Analysis System) program. The mean and coefficient of variation for the age-atfirst-calving was $1252.77 \pm 125$ days (10.41 months; $10.56 \%$ ) and, for the calving interval, values were $591.73 \pm 149.91$ days (12.49 months; 38.74\%). Uncharacteristic analyses were used to estimate genetic parameters for heritability values, obtaining values of approximately 0.026 and 0.006 for IPP and IDP, respectively. The repeatability estimate for the IDP characteristic, as calculated by uncharacteristic analysis, had a generally low 
Rev. Bras. Saúde Prod. Anim., Salvador, v.16, n.2, p.290-299 abr./jun.., 2015 http://www.rbspa.ufba.br ISSN 15199940

value of 0.025 . Similarly, for trait analysis, heritability values were 0.03 and 0.06 for IPP and IDP, respectively, and the estimated repeatability coefficient was 0.14 . The genetic correlations between the reproductive characteristics (IPP and IDP) observed in this study were equal to 0.06 . The reproductive performance of the herd studied, in tropical climate conditions, can be considered to be satisfactory, in accordance with the parameters obtained by other authors in different regions.

Keywords: beef cattle, genetic correlation, reproductive performance

\section{INTRODUÇÃO}

O Brasil detém o maior rebanho bovino comercial do mundo e é o segundo maior produtor de carne bovina, com 6,7 milhões de toneladas, exportando sua produção para mais de 170 países (ANUALPEC, 2009; ABIEC, 2010).

Apesar de ser destaque na produção de carne bovina, o rebanho brasileiro ainda apresenta desempenho reprodutivo aquém do ideal, o que resulta em baixa eficiência produtiva. Segundo Abreu et al. (2006) a taxa média de natalidade nos rebanhos de corte do Brasil Central ainda não atinge o patamar de $60 \%$ e o intervalo de partos em condições normais varia de 14 a 18 meses, visto que, o intervalo de partos ideal é de 12 meses.

O aumento da eficiência econômica na bovinocultura está relacionado diretamente com a melhoria na eficiência reprodutiva (ANDRÉA et al., 2007; LÔBO et al., 2008; BALDI et al., 2008). A busca por essa eficiência em programas de melhoramento genético tem-se voltado para a seleção de características que determinam aumento da fertilidade dos animais, como incrementos nas taxas de precocidade sexual e parição (PEROTTO et al., 2006). Portanto, é de suma importância avaliar $o$ potencial genético dos rebanhos bovino para que se possa, através da seleção dos animais geneticamente superiores, agregar valor às características que compõem os índices reprodutivos.

Além disso, a influência de fatores não genéticos, como os ambientais, sobre as características reprodutivas deve ser estudada, pois estes podem intervir no desenvolvimento sexual dos bovinos, sendo relevante no favorecimento do melhoramento genético efetivo dessas caraterísticas (SILVEIRA et al., 2004).

Objetivou-se avaliar a influência de alguns fatores ambientais e estimar os parâmetros genéticos por meio da utilização de modelo animal uni e bicaracterística para as características reprodutivas, idade ao primeiro parto e intervalo de partos de fêmeas da raça Nelore nos Estados do Maranhão e Piauí.

\section{MATERIAL E MÉTODOS}

Os dados das características reprodutivas de fêmeas da raça Nelore, analisados neste trabalho, foram obtidos junto à Associação Brasileira de Criadores de Zebu (ABCZ), com animais nascidos no período de 1976 a 2012, incluídos no sistema de controle reprodutivo realizado pela $\mathrm{ABCZ}$, nos estados do Maranhão e Piauí que formam a sub-região denominada MeioNorte. É entendida como uma área de passagem entre o sertão semi-árido (Piauí) e a Amazônia (oeste Maranhense). Pela classificação de Köppen o clima predominante é o de inverno seco e verão chuvoso com precipitação pluviométrica anual média de $1.365 \mathrm{~mm}$. Apresenta vegetação abundante de matas de babaçu e carnaúba (IBGE, 2010). Foram realizadas algumas restrições no banco 
Rev. Bras. Saúde Prod. Anim., Salvador, v.16, n.2, p.290-299 abr./jun.., 2015 http://www.rbspa.ufba.br ISSN 15199940

de dados para adequar possíveis distorções existentes. Estas restrições se referem às seguintes informações: identificador do animal, data de nascimento do animal, identificador do pai, identificador da mãe, data de nascimento do pai, data de nascimento da mãe; raça do animal, raça do pai, raça da mãe; categoria de registro do animal, categoria de registro do pai e categoria de registro da mãe.
Inicialmente os dados foram submetidos a uma prévia análise de consistência. Foram utilizados animais com idade de 03 desvios-padrão em relação à média de idade dos animais de mesmo grupo de contemporâneos, reprodutores com pelo menos três filhos em cada estado, grupos de contemporâneos com no mínimo três animais e vacas com idades inferiores há 20 anos (Tabela 1).

Tabela 1. Composição do arquivo de dados avaliados das caraterísticas idade ao primeiro parto (IPP) e intervalo de partos (IDP) de fềmeas da raça Nelore

\begin{tabular}{lc}
\hline \multicolumn{2}{c}{ Dados da característica IPP } \\
\hline Total de registros & 14674 \\
Número de fêmeas utilizadas & 1799 \\
Animais na matriz de parentesco & 6502 \\
Grupos contemporâneos & 108 \\
\hline \multicolumn{2}{l}{ Dados da característica IDP } \\
\hline Total de registros & 14765 \\
Número de fêmeas utilizadas & 1231 \\
Animais na matriz de parentesco & 4510 \\
Grupos contemporâneos & 87 \\
\hline
\end{tabular}

Para o estudo dos efeitos ambientais foi utilizado o procedimento General Linear Models (GLM) do programa
Statistical Analysis System (SAS, 2003), segundo o modelo estatístico abaixo:

$$
y_{i j k i m o}=\mu+E_{j}+F E_{j k}+A N_{i}+M N_{m}+S_{n}+e_{i j k i m n o}
$$

onde: $Y_{i j k l m n o}=$ Idade ao primeiro parto (IPP) e intervalo de partos (IDP); $\mu=$ constante inerente a todas as observações; $E_{j}=$ efeito fixo do Estado $\mathrm{j} ; F E_{J k}=$ efeito fixo da estação (IPP) e (IDP); $A N_{l}=$ efeito fixo do ano de parição $1 ; M N_{m}=$ efeito fixo do criador $\mathrm{m} ; S_{n}=$ efeito fixo do sexo da cria $\mathrm{n}$; $e_{i j k l m \text { по }}=$ erro aleatório independentemente distribuído com média zero e variância $\sigma^{2}$.

As análises genéticas para estimar os componentes de variância genética aditiva e os parâmetros genéticos para a idade a primeira parição (IPP) e intervalo de partos (IDP) foram executados por meio de modelo animal, em análise uni e bicaracterística, são: Modelo 1 (análise unicaracterística): $y=X b+Z a+e$, e Modelo 2 (análise bicaracterística):

$y=X b+Z a+W c+e_{j} . \quad E m$ análise multicaracterística o modelo completo em notação matricial é dado como segue: 


$$
\left[\begin{array}{l}
y_{1} \\
y_{2}
\end{array}\right]=\left[\begin{array}{cc}
X_{1} & 0 \\
0 & X_{2}
\end{array}\right]\left[\begin{array}{l}
b_{1} \\
b_{2}
\end{array}\right]+\left[\begin{array}{cc}
Z_{1} & 0 \\
0 & Z_{2}
\end{array}\right]\left[\begin{array}{l}
a_{1} \\
a_{2}
\end{array}\right]+\left[\begin{array}{cc}
W_{1} & 0 \\
0 & W_{2}
\end{array}\right]\left[\begin{array}{l}
c_{1} \\
c_{2}
\end{array}\right]+\left[\begin{array}{l}
e_{1} \\
e_{2}
\end{array}\right]
$$

em que $y$ é o vetor das observações da característica medida nos animais (IPP e IDP); $b$ é o vetor de efeitos "fixos"; $a$ é o vetor de efeitos aleatórios que representa os valores genéticos aditivos diretos de cada animal; $c$ é o vetor de efeitos de ambiente permanente (não correlacionado) para a característica IDP; $e$ é o vetor de erros aleatórios; $X$, $Z$ e $W$ são matrizes de incidência que relacionam as observações aos efeitos fixos, ao efeito aleatório aditivo direto e de ambiente permanente, respectivamente.

Admitindo-se que $y, a, c$ e $e$ tenham distribuição normal multivariada, temse $\quad$ que: $\quad \sigma_{a}^{2}=A \sigma_{a}^{2} ; \quad \sigma_{a}^{2}=I \sigma_{o}^{2}$; $\sigma_{g}^{2}=I \sigma_{g}^{2}=R$ e; $\sigma_{y}^{2}=Z G Z^{\prime} \sigma_{a}^{2}+W I \sigma_{o}^{2} W^{\prime}+R$, sendo: $G=A \otimes G_{0}, R=I \otimes R_{0}$, Onde: $A=$ matriz de parentesco, $n x n$, do numerador do coeficiente de parentesco entre os $n$ animais; $G_{0}$ e $R_{0}=$ matrizes, $q \times q$, de (co) variâncias genéticas diretas e variâncias residuais, respectivamente, para cada característica; $I=$ matriz identidade; $\otimes=$ operador produto-direto. Assim, a matriz de covariância genética, $G_{0}=\left[\begin{array}{ll}\sigma_{a 11}^{2} & \sigma_{a 12}^{2} \\ \sigma_{a 21}^{2} & \sigma_{a 22}^{2}\end{array}\right]$, em que: $\sigma_{a i i}^{2}=$ variância genética aditiva da característica $\mathrm{i} ; \quad \sigma_{\text {aij }}^{2}=$ covariância genética aditiva entre as características $\mathrm{i}$ e $\mathrm{j} ; \quad \sigma_{\theta i t}^{2}=$ variância residual da característica $\mathrm{i}$.

Os efeitos fixos considerados no modelo foram grupo contemporâneo e idade da matriz ao parto, como covariável linear e quadrática. Os grupos de contemporâneos foram definidos com animais pertencentes ao mesmo estado, fazenda, ano de nascimento e mês de nascimento.

As estimativas dos componentes de variância e covariância genética e os parâmetros genéticos (herdabilidades e correlações) foram obtidos com auxílio do programa WOMBAT (MEYER, 2006) que utiliza o Método da Máxima Verossimilhança Restrita, por meio do algoritmo Maximização da Esperança (EM), para maximização da função de verossimilhança. Este procedimento é muito estável numericamente e apresenta convergência mesmo que os valores iniciais não estejam próximos dos valores paramétricos. O critério de convergência utilizado foi $10^{-8}$.

\section{RESULTADOS E DISCUSSÃO}

Na tabela 2 estão expostos os valores dos seguintes dados estatísticos: desvios padrão; valores mínimo e máximo; número total de observações e de grupo de contemporâneos para as características de IPP e IDP. As médias obtidas e os coeficientes de variação para característica de idade ao primeiro parto foram $1252,77 \pm 125$ dias $(41,76$ meses) a $10,56 \%$, mostrando que as novilhas foram cobertas pela primeira vez com uma idade aproximada de três anos e meio. Resultado semelhante foi apresentado em estudo desenvolvido por Azevêdo et al. (2006), que foi de 45,14 meses para esta característica. As médias encontradas para a característica intervalo de parto foram $591,729 \pm 149,91$ dias $(19,72$ meses $)$ a $38,74 \%$, respectivamente. Segundo a literatura, em diversos estudos realizados com bovinos de corte, os 
Rev. Bras. Saúde Prod. Anim., Salvador, v.16, n.2, p.290-299 abr./jun.., 2015 http://www.rbspa.ufba.br ISSN 15199940

resultados de IDP apresentam uma duração média de alta variação, sendo o intervalo de 12 meses considerado o ideal. Neste trabalho o resultado obtido (19,72 meses) foi acima do desejável, pois este período aumenta o tempo de recuperação do sistema reprodutivo da vaca para que a mesma possa ter uma nova gestação, diminuindo assim, o número de crias produzidas por matriz durante sua vida produtiva.

Tabela 2. Número de observações $(\mathrm{N})$, médias, desvio padrão e valores mínimos e máximos para as características de idade ao primeiro parto (IPP) e intervalo de parto (IDP) em fêmeas da Raça Nelore

\begin{tabular}{lccccccc}
\hline Características & $\mathrm{N}$ & Média & DP & CV & Mín. & Máx. & GC \\
\hline IPP & 8301 & 1252,77 & 132,309 & 10,56 & 1050,00 & 1500,00 & 1738 \\
IDP & 14765 & 591,729 & 229,250 & 38,74 & 365,00 & 1799,00 & 1847 \\
\hline
\end{tabular}

$\mathrm{DP}=$ desvio padrão; Mín.= mínimo; Máx. $=$ máximo; $\mathrm{CV}=$ coeficiente de variação; $\mathrm{GC}=$ grupo de contemporâneos.

A média para IPP observada foi de 1252,77 dias, e superior à relatada por Dias et al. (2004) de 1033,15 dias e Laureano et al. (2011) de 1052,90 dias, ambos trabalhando com novilhas da Raça Nelore. Quanto à característica de IDP o valor encontrado (19,72 meses), neste trabalho, foi superior ao valor (13,14 meses) encontrado por Souza et al. (1995).

Vale ressaltar que precocidade ao primeiro parto e baixo intervalo de partos são relevantes do ponto de vista reprodutivo, pois a eficiência reprodutiva dos animais está ligada diretamente ao bom manejo na fase de crescimento, gerando coberturas e partos precoces com aproveitamento pleno do animal na sua vida útil.

No que se refere à estimação de componentes de variância pelo modelo unicaracterística, observou-se que o coeficiente de variação foi baixo, demonstrando que a amostra foi suficiente para obter valores representativos da população para as estimativas a posteriori das distribuições dos componentes de (co) variância (Tabela 3). As estimativas dos componentes de variância e parâmetros genéticos obtidos permitiram a obtenção das estimativas dos componentes de (co) variância das características estudadas IPP e IDP, dos parâmetros genéticos: herdabilidade, repetibilidade e correlações genéticas entre as características.

$\mathrm{O}$ resultado da variância genética aditiva direta encontrado para a característica IPP $(337,1)$ foi inferior ao encontrado por Laureano et al. (2011), que foi de 731,47. Segundo estes autores, o baixo valor apresentado para esta variância em animais zebuínos, pode ser explicado pela adoção de estação de monta de curto período e retardo em expor as novilhas à monta ou inseminação artificial.

No âmbito da pesquisa, os resultados encontrados para os valores de herdabilidade para as características de IPP e IDP, neste estudo $(0,026$ e 0,06 , respectivamente) (Tabela 3), são próximos aos relatados por Boligon et al. (2008) com raça Nelore, relatando estimativas de herdabilidade para IPP e IDP praticamente nulas, 0,01 e 0,02, respectivamente. Resultado superior foi verificado por Pereira et al. (2010) no 
Rev. Bras. Saúde Prod. Anim., Salvador, v.16, n.2, p.290-299 abr./jun.., 2015 http://www.rbspa.ufba.br ISSN 15199940

qual o valor de herdabilidade para característica IPP foi de 0,05 .

Segundo Boligon et al. (2010) a baixa herdabilidade encontrada na característica IPP sugere que o uso da mesma como critério no processo de seleção promoverá baixo ganho genético, no que tange a entrada das fêmeas Nelore à vida reprodutiva.

Tabela 3. Médias a posteriori dos componentes de (co) variância e parâmetros genéticos em análise Unicaracterística

\begin{tabular}{lcccccc}
\hline Características & $\boldsymbol{\sigma}_{a}^{2}$ & $\boldsymbol{\sigma}_{c}^{2}$ & $\boldsymbol{\sigma}_{e}^{2}$ & $\boldsymbol{\sigma}_{p}^{2}$ & $\boldsymbol{h}^{2}$ & $\boldsymbol{t}$ \\
\hline IPP & 337,1 & - & 12457 & 12784 & 0,026 & - \\
IDP & 286,3 & 1099,6 & 41824 & 43210 & 0,006 & 0,025 \\
\hline
\end{tabular}

IPP $=$ Idade ao primeiro parto; IDP $=$ Intervalo de parto; $\sigma_{a}^{2}=$ variância devido ao efeito genético aditivo direto; $\sigma_{g}^{2}=$ variância devido ao efeito de ambiente permanente; $\sigma_{g}^{2}=$ variância residual; $\sigma_{p}^{2}=$ variância fenotípica; $h^{2}=$ herdabilidade da característica; $t=$ repetibilidade da característica.

É importante ressaltar que diferentes amostras e modelos podem causar disparidades nas estimativas de parâmetros genéticos para essas determinadas características reprodutivas, isso ocorre porque a IPP é bastante dependente do manejo reprodutivo e da idade em que as fêmeas são expostas à concepção, dependendo da forma que tais efeitos são considerados ou não nos modelos, essas estimativas obtidas podem sofrer variações, conforme detectado neste presente trabalho.

Algumas herdabilidades para características de IPP em diferentes modelos uni e bicaracterísticas são consideradas baixas, devido a uma porção da variação existente na característica não ser estimada. Dessa forma, mesmo utilizando-se de um arquivo de genealogia bastante completo, às vezes não é possível obter uma real variabilidade genética existente na população (BOLIGON et al., 2008).

A estimativa de repetibilidade para a característica IDP em analise unicaracterística apresentou-se, de forma geral, com baixa magnitude 0,025, de modo que serão necessárias mais mensurações nos animais para representar sua real capacidade de reprodução, ou seja, uma única observação feita no indivíduo apresenta pequeno valor para a sua seleção, havendo necessidade de que novas ocorrências sejam consideradas (Tabela 3). Destaca-se que com o aumento de mensurações nos animais pode-se minimizar os possíveis erros decorrentes da coleta dos dados. Resultado superior de repetibilidade para esta característica foi encontrado por Facó et al. (2008) de 0,09 .

$\mathrm{Na}$ mesma ordem, para análise bicaracterística, as herdabilidades foram de 0,03 e 0,06 para IPP e IDP, respectivamente (Tabela 4). Dessa forma, é fácil observar que houve uma sensível melhora nas estimativas e um incremento nos componentes de variância. Assim as características que se repetem ao longo da vida do animal, a exemplo do IDP, se expressam em diferentes intensidades em cada ocorrência, e o coeficiente de repetibilidade estimado de 0,14, respectivamente. Apesar de baixo, este coeficiente mostra-se como importante ferramenta quando se trabalha com 
Rev. Bras. Saúde Prod. Anim., Salvador, v.16, n.2, p.290-299 abr./jun.., 2015 http://www.rbspa.ufba.br

animais que apresentam várias medidas da mesma característica, o que pode auxiliar a predição de desempenhos futuros dos animais.

A baixa variância genética aditiva indica que a seleção dos melhores animais pode não ser satisfatória para a melhoria dessas características no rebanho, visto que, o efeito ambiental tem grande influencia na variação dessas características. Dessa forma, medidas de manejo mais favoráveis devem melhorar as características reprodutivas reduzindo as taxas de IPP e IDP nos rebanhos avaliados.

Tabela 4. Médias a posteriori dos componentes de (co) variância e parâmetros genéticos em análise bicaracterística

\begin{tabular}{lrlllllllll}
\hline Características & $\sigma_{a}^{2}$ & $\sigma_{a x, y}$ & $\boldsymbol{\sigma}_{c}^{2}$ & $\boldsymbol{\sigma}_{\mathrm{e}}^{2}$ & $\boldsymbol{\sigma}_{\boldsymbol{\varepsilon} x, y}$ & $\boldsymbol{\sigma}_{\vec{p}}^{2}$ & $\boldsymbol{\sigma}_{p x, y}$ & $\boldsymbol{h}^{2}$ & $\boldsymbol{r}$ & $\boldsymbol{t}$ \\
\hline IPP & 496,5 & & & 12183 & & 1268 & & 0,03 & & \\
& & 80,46 & & & $-2940,8$ & & $-2860,3$ & & 0,06 & \\
IDP & 2869,6 & & 3690,5 & 39970 & & 4653 & & 0,06 & & 0,14 \\
\hline
\end{tabular}

IPP = idade ao primeiro parto; IDP = intervalo de parto; $\sigma_{a}^{2}=$ variância devido ao efeito genético aditivo direto; $\sigma_{\alpha x, y}=$ covariância genética entre a primeira e a segunda característica de cada análise; $\sigma_{\varepsilon}^{2}=$ variância devido ao efeito de ambiente permanente; $\sigma_{\vartheta}^{2}=$ variância residual; $\sigma_{\nexists x y}=$ covariância genética entre a primeira e a segunda característica de cada análise; $\sigma_{p}^{2}=$ variância fenotípica; $\sigma_{p} x, y=$ covariância fenotípica entre a primeira e a segunda característica de cada análise; $h^{2}=$ herdabilidade da característica; $r=$ correlação genética entre a primeira e a segunda característica de cada análise; $t=$ repetibilidade da característica.

As correlações genéticas entre as características de reprodução observadas neste trabalho, referentes às características reprodutivas entre IPP e IDP, foram iguais a 0,06 sugerindo que a seleção para o aumento de uma acarretará em melhoria da outra, por intermédio de seleção indireta.

Estudando um rebanho da raça Nelore no Estado do Maranhão, Cavalcante et al. (2000) analisou 587 dados e observaram média do intervalo de parto de 431,83 $\pm 142,76$ dias (14,16 meses), em regime de pasto. Estes autores verificaram que $o$ ano do parto influenciou o intervalo de parto, porém, esta tendência não foi observada para o mês do parto e idade da vaca ao parto.

Da mesma forma, Silveira et al. (2004) analisando 3.069 dados de animais da raça Nelore, criados em regime de pasto, no Estado do Mato Grosso do Sul, observaram uma média de 465 dias (15,24 meses) para o intervalo de parto. Verificou-se efeito da ordem de parição, mês e ano de nascimento do bezerro sobre o intervalo de parto, porém, o sexo da cria não apresentou esta tendência. $\mathrm{O}$ efeito do ano sobre esta característica pode ser explicado pela má distribuição de chuva durante o ano, contribuindo para ocorrência de um longo período de déficit hídrico, escassez de forragem e consequente prejuízo para recuperação do estresse do parto pelas matrizes.

Como relatado por Azevêdo et al. (2006) e Oliveira et al. (2007) a baixa herdabilidade nos rebanhos indica menor variabilidade genética aditiva na expressão do IDP, sendo esta característica bastante influenciada 
Rev. Bras. Saúde Prod. Anim., Salvador, v.16, n.2, p.290-299 abr./jun.., 2015 http://www.rbspa.ufba.br ISSN 15199940

pelas condições de ambiente. O IDP nestes rebanhos pode ser mais fácil $\mathrm{e}$ rapidamente manipulado através da implantação de medidas de manejo mais adequadas, haja vista que o ambiente seria o fator de maior relevância para a obtenção de mudanças positivas na expressão dessa característica.

$\mathrm{O}$ presente estudo revelou que as médias para IPP e IDP mostraram-se elevadas nos rebanhos avaliados, o que compromete a atividade reprodutiva. As herdabilidades observadas apesar de serem baixas foram condizentes com os resultados encontrados na literatura.

A adoção de um manejo reprodutivo adequado com base no registro de todas as informações inerentes à reprodução, como o escore de condição corporal das novilhas e vacas antes de entrarem na estação de monta, a apresentação dos sinais de cio, data de cobertura e/ou inseminação artificial, presença de partos distócicos, abortos, etc., contribuirá com o desempenho reprodutivo do rebanho, de forma a evitar, por exemplo, que o intervalo de partos aumente e onere o custo de produção com a manutenção no rebanho de uma matriz com menor número de concepções, o que refletirá no baixo aproveitamento na produção de leite.

Para a melhoria nos índices da caraterística de idade ao primeiro parto, selecionar e eliminar do rebanho, novilhas que desmamem com baixo peso o produto de sua primeira concepção, pode ser uma alternativa viável para aumentar o peso dos produtos desmamados.

\section{AGRADECIMENTOS}

À Associação Brasileira de Criadores de ZebuABCZ, ao Dr. Rodrigo Junqueira Pereira, pela concessão dos dados que compõe este trabalho $e$ à Coordenação de Aperfeiçoamento de
Pessoal de Nivel Superior - CAPES, pela concessão de bolsa de estudos.

\section{REFERÊNCIAS}

ASSOCIAÇÃO BRASILEIRA DAS INDÚSTRIAS EXPORTADORAS DE CARNE - ABIEC. Brasil lidera ranking de exportação de carne bovina. Disponível em:

$<$ http://www.abiec.com.br/news_view.a sp?id=\%7BCAACE975-B5D1-43379F3B-580E7118CB45\%7D>. Acesso em: 18 abr. 2010.

ABREU, U.G.P.; LOPES, P.S.; TORRES, R.A.; SANTOS, H.N. Avaliação da introdução de tecnologias no sistema de produção de gado de corte no Pantanal. Desempenho e descarte de matrizes. Revista

Brasileira de Zootecnia, v.35, n.6, p.2496-2503, 2006.

ANDRÉA, M.V.; MEIRELLES, F.V.; LÔBO, R.B.; MILLAZZOTTO, M.P.; MARSON, E.P.; VILA, R.A.; BITTENCOURT, T.C.B. dos S.C, de; MARCONDES, C.R. Polimorfismo SSCP e a puberdade em novilhas da raça Nelore. Magistra, v.19, n.3, p.198203, 2007.

ANUALPEC. Anuário da Pecuária Brasileira. São Paulo: Instituto FNP, 2009.

AZEVÊDO, D.M.M.R.; MARTINS

FILHO, R.; LÔBO, R.N.B.;

MALHADO, C.H.M.; LÔBO, R.B.; MOURA, A.D.A.A.; PIMENTA FILHO, E.C. Desempenho reprodutivo de vacas Nelore no Norte e Nordeste do Brasil. Revista Brasileira de

Zootecnia, v.35, n.3, p.988-996, 2006. 
Rev. Bras. Saúde Prod. Anim., Salvador, v.16, n.2, p.290-299 abr./jun.., 2015 http://www.rbspa.ufba.br ISSN 15199940

BALDI, F.; ALENCAR, M.M.; FREITAS, A.R.; BARBOSA, R.T. Parâmetros genéticos para característica de tamanho e condição corporal, eficiência reprodutiva e longevidade em fêmeas da raça Canchim. Revista Brasileira de Zootecnia, v.37, n.2, p.247-253, 2008.

BOLIGON, A.A.; VOZZI, P.A.; NOMELINI, J.; RORATO, P.R.N.; BEZERRA, L.A.F.; LÔBO, R.B. Parâmetros genéticos para idade ao primeiro parto estimados por diferentes modelos para rebanhos da raça Nelore.

Revista Ciência Rural, v.38, n.2, p.432436, 2008.

BOLIGON, A.A.; ALBUQUERQUE, L.G. Correlações genéticas entre escores visuais e características reprodutivas em bovinos Nelore usando inferência bayesiana. Pesquisa Agropecuária Brasileira, v.45, n.12, p.1412-1418, 2010.

CAVALCANTE, F.A.; MARTINS FILHO, R.; CAMPELLO, C.C.; LOBO, R.N.B.; MARTINS, G.A. Intervalo de partos em rebanho Nelore na Amazônia Oriental. Revista Brasileira de

Zootecnia, v.29, n.5, p.1327-1331, 2000.

DIAS, L.T.; FARO, L.EL.;

ALBUQUERQUE, L.G. Estimativas de herdabilidade para idade ao primeiro parto de novilhas da raça Nelore. Revista Brasileira de Zootecnia, v.33, n.1, p.97102, 2004.

FACÓ, O.; LÔBO, R.N.B.; MARTINS

FILHO, R.; MARTINS, G.A.; OLIVEIRA, S.M.P.; AZEVÊDO, D.M.M.R. Efeitos genéticos aditivos e não-aditivos para características produtivas e reprodutivas em vacas mestiças Holandês $\times$ Gir. Revista

Brasileira de Zootecnia, v.37, n.1, p.4853, 2008.
INSTITUTO BRASILEIRO DE GEOGRAFIA E ESTATÍSTICA - IBGE. Disponível em:

$<$ http://www.ibge.gov.br $>$. Acesso em: 20 maio 2010.

LAUREANO, M.M.M.; BOLIGON, A.A.; COSTA, R.B.; FORNI, S.; SEVERO, J.L.P.; ALBUQUERQUE, L.G. Estimativas de herdabilidade e tendências genéticas para características de crescimento e reprodutivas em bovinos da raça Nelore. Arquivo Brasileiro de Medicina Veterinária e Zootecnia, v.63, n.1, p.143-152, 2011.

LÔBO, R.B.; BEZERRA, L.A.F.; FARIA, C.U.; MAGNABOSCO, C.U.; ALBUQUERQUE, L.G.; BERGMANN, J.A.G.; SAINZ, R.D.; OLIVEIRA, H.N. Avaliação genética de touros e matrizes da raça nelore: sumário 2008. 14.ed. Ribeirão Preto: ANCP, 2008. 124p.

MEYER, K. "WOMBAT" - Digging deep for quantitative genetic analyses by restricted maximum likelihood. In: WORLD CONGRESS OF GENETICS, 8., 2006, Belo Horizonte. Proceedings... Belo Horizonte, 2006.

OLIVEIRA, M.M.; ROTA, E.L.; DIONELLO, N.J.L.; AITA, M.F. Herdabilidade e correlações genéticas do perímetro escrotal e idade ao primeiro parto com características produtivas em bovinos de corte: Revisão. Revista Brasileira de Agrociência, v.13, n.2, p.141-146, 2007.

PEREIRA, M.C.; YOKOO, M.J.; BIGNARDI, A.B.; SEZANA, J.C.; ALBUQUERQUE, L.G. Altura da garupa e sua associação com características reprodutivas e de crescimento na raça Nelore. Pesquisa Agropecuária Brasileira, v.45, n.6, p.613-620, 2010. 
Rev. Bras. Saúde Prod. Anim., Salvador, v.16, n.2, p.290-299 abr./jun.., 2015 http://www.rbspa.ufba.br

PEROTTO, D.; ABRAHÃO, J.J.S.;

KROETZ, I.A. Intervalo de partos de fêmeas bovinas Nelore, Guzerá x

Nelore, Red Angus x Nelore,

Marchigiana x Nelore e Simental x

Nelore. Revista Brasileira de

Zootecnia, v.35, n.3, p.733-741, 2006.

STATISTICAL ANALYSIS SYSTEM

- SAS. User's guide. Cary, NC: SAS

Institute, 2003. 129p.

SILVEIRA, J.C.; McMANUS, C.;

MASCIOLI, A.S.; SILVA, L.O.C.;

SILVEIRA, A.C.; GARCIA, J.A.S.;

LOUVANDINI, H. Fatores Ambientais

e Parâmetros Genéticos para

Características Produtivas e

Reprodutivas em um Rebanho Nelore no Estado do Mato Grosso do Sul.

Revista Brasileira de Zootecnia, v.33, n.6, p.1432-1444, 2004.

SOUZA, J.C.; RAMOS, A.A.; FERRAZ FILHO, P.B. Estudo dos intervalos de partos de matrizes da Raça Nelore. Revista Brasileira de

Zootecnia, v.24, n.4, p.645-651, 1995.

Data recebimento: $27 / 03 / 2014$

Data de aprovação: 16/03/2015 